\title{
Non-minimally Coupled Tachyon Field in Teleparallel Gravity
}

\author{
Behnaz Fazlpour $^{a}$ 1] and Ali Banijamali ${ }^{b} 2$ \\ ${ }^{a}$ Department of Physics, Babol Branch, Islamic Azad University, Babol, Iran \\ ${ }^{b}$ Department of Basic Sciences, Babol University of Technology, Babol, Iran
}

\begin{abstract}
We perform a full investigation on dynamics of a new dark energy model in which the four-derivative of a non-canonical scalar field (tachyon) is non-minimally coupled to the vector torsion. Our analysis is done in the framework of teleparallel equivalent of general relativity which is based on torsion instead of curvature. We show that in our model there exists a late-time scaling attractor (point $P_{4}$ ), corresponding to an accelerating universe with the property that dark energy and dark matter densities are of the same order. Such a point can help to alleviate the cosmological coincidence problem. Existence of this point is the most significant difference between our model and another model in which a canonical scalar field (quintessence) is used instead of tachyon field.
\end{abstract}

PACS numbers: 95.36.+x, 98.80.-k, 04.50.kd

Keywords: Tachyon field; Teleparallel gravity; Non-minimal coupling.

\footnotetext{
${ }^{1}$ b.fazlpour@umz.ac.ir

2a.banijamali@nit.ac.ir
} 


\section{Introduction}

A mysterious component of our universe with negative pressure can be a reasonable description for one of the most important discoveries in cosmology. This component is known as dark energy (for reviews on dark energy see $[1,2]$ and references therein) and the discovery is the universe late-times acceleration supported by the cosmological observations [3].

Besides the cosmological constant that suffers from cosmological constant problem [4], the simplest candidate of dark energy is a canonical scalar field, the so-called quintessence [5]. One can also generalize quintessence model by including a non-minimal coupling between scalar field and gravity [6-8]. Non-canonical scalar field models of dark energy [9-13] and specially tachyon field model [14, 15] also exist in the literature notably. Furthermore, teleparallel gravity originally proposed by Einstein [16, 17] is a theory of gravity which is based on torsion instead of curvature formulation. In teleparallel gravity the curvature-less Weitzenbock connection is used rather than the torsion-less Levi-Civita one [16-19] and dynamical objects are the four linearly independent vierbein (tetrad) fields. The teleparallel Lagrangian is described by torsion scalar $T$. In Friedmann-Robertson-Walker (FRW) space-time it is completely equivalent to a matter dominated universe in the framework of general relativity. Thus, teleparallel gravity without modification can not describe the accelerated expansion of the universe. Inspired by the similar procedures in general relativity there are two ways for such modification. The first one is the construction of $f(T)$ gravity by extending $T$ to an arbitrary function [20-23] and the second one is to directly add dark energy into teleparallel gravity allowing also a non-minimal coupling between dark energy and gravity. Note, however that in $f(T)$ theories the action and field equations are not invariant under local Lorentz transformations. This problem along with the appearance of extra degrees of freedom with respect to general relativity are crucial issues of $f(T)$ gravity [23]. Recently a non-minimally coupled quintessence field in the framework of teleparallel gravity, the so-called teleparallel dark energy model, has been considered [24-28] and its generalization to a non-canonical scalar field (tachyon) model has been studied in [29, 30]. In these models scalar field non-minimally coupled to torsion scalar. As it was emphasized in [24] replacing the Ricci scalar $R$ by torsion scalar $T$ in the case of the minimal coupling of scalar field dark energy models has no new result in the level of field equations and perturbation as well. However, things are different if we switch on the non-minimal coupling. In this case the resulting coupled equations do not coincide. Clearly, teleparallel gravity under the non-minimal coupling is a different theory.

In our previous works $[46,47]$ we have investigated dynamical analysis of non-interacting and interacting tachyonic teleparallel dark energy in details. In [46] we have extracted critical points of the non-interacting scenario where they are all dark energy dominated solutions. When interaction between dark energy and dark matter was considered in the model, although late-time accelerated attractor solutions have been found, there was no scaling attractor [47].

Very recently inspired by the fact that in teleparallel gravity a vector field interacts with the vector part of the torsion [31, 32], Otalora has proposed a new dark energy model in which the four-derivative (which is a vector field) of a canonical scalar field (quintessence) couples non-minimally to the vector part of torsion [33]. The author has studied dynamics of the model and its cosmological implications in details. Here we generalize such a model using tachyon field as a responsible for dark energy.

The plan of the work is the following: In section 2 we present the model and derive basic equations and expressions including energy density, pressure and equation of motion of the scalar field. In section 3 dynamical system study of the model is done and the cosmological implications are discussed. Section 4 is devoted to our conclusions.

\section{Tachyon Field in Teleparallel Gravity: Basic Equations}

Three components of torsion tensor $T_{\mu \nu}^{\lambda}$, namely vector torsion, axial torsion and pure tensor are given as follows respectively [33]

$$
\mathcal{V}_{\mu}=T_{\nu \mu}^{\nu}
$$




$$
\begin{gathered}
\mathcal{A}^{\mu}=\frac{1}{6} \epsilon^{\mu \nu \rho \sigma} T_{\nu \rho \sigma}, \\
\mathcal{T}_{\lambda \mu \nu}=\frac{1}{2}\left(T_{\lambda \mu \nu}+T_{\mu \lambda \nu}\right)+\frac{1}{6}\left(g_{\nu \lambda} \mathcal{V}_{\mu}+g_{\nu \mu} \mathcal{V}_{\lambda}\right)-\frac{1}{3} g_{\lambda \mu} \mathcal{V}_{\nu}
\end{gathered}
$$

Our model with a non-minimal coupling between four-derivative of tachyon field and vector torsion (1) can be described by the following action

$$
S=\int d^{4} x h\left[\frac{T}{2 \kappa^{2}}-V(\varphi) \sqrt{1-\partial_{\mu} \varphi \partial^{\mu} \varphi}+\eta f(\varphi) \partial_{\mu} \varphi \mathcal{V}^{\mu}\right]+S_{m},
$$

where $h \equiv \operatorname{det}\left(h^{a}{ }_{\mu}\right)=\sqrt{-g}\left(h^{a}{ }_{\mu}\right.$ are the orthonormal tetrad components), $T$ is the torsion scalar corresponding to teleparallel equivalent of general relativity (TEGR) and $\kappa^{2}=8 \pi G$ while $G$ is a bare gravitational constant (for reviews on teleparallelism see [31, 32]). The second part in the action (4) is the relevant Lagrangian of the tachyon field and $S_{m}\left(\psi_{m}, h^{a}{ }_{\rho}\right)$ is the action of the matter field which is chosen as the cold dark matter (DM). Also $\eta$ is a dimensionless constant measuring the non-minimal coupling and $f(\varphi)$ is an arbitrary function of the scalar field. In the other hand, note that there is no interaction between dark matter and dark energy.

We mention that the model (4) is a generalization of the model proposed in [33] by using a non-canonical scalar field (tachyon) instead of canonical scalar field (quintessence). Tachyon field originates from a fundamental theory such as string theory and has interesting cosmological implications (see for example [14, 15]). In the context of general relativity, non-minimal couplings are generated by quantum corrections to the scalar field theory and they are essential for the renormalizability of the scalar field theory in curved space (see [39] and references therein). In the other side, non-minimal coupling between derivatives of a scalar field and curvature may appear in some Kaluza-Klein theories [40, 41].

The gravitational coupling of the fundamental fields in teleparallel gravity is a very controversial subject. Since there is no experimental data to help us, we should rely on equivalence between general relativity and teleparallel gravity. According to this formulation, each one of the fundamental fields of nature (scalar, spinor, and electromagnetic) are required to couple to torsion in a such a way to preserve the equivalence between teleparallel gravity and general relativity [31]. It is shown in [32] that in the context of teleparallel gravity a scalar field by itself does not feel gravity but its four-derivative (which is a vector field) interacts with the vector part of torsion.

As it is noted in [33] the model in (4) and tachyonic teleparallel dark energy model [29] in which tachyon field non-minimally coupled to torsion scalar instead of vector torsion, are mathematically related through a conformal transformation although they are physically different.

In the other side, the possibility that the dynamic Poincare gauge theory connection, reflected in dynamic Poincare gauge theory torsion, provides the accelerating force in the universe has been explored by Shie et al. in [38]. The scalar mode torsion in the model plays the role of the imperceptible dark energy and it is naturally obtained from the geometry of the Riemann-Cartan space-time, instead of from an exotic scalar field or a designed mechanism. Note however, as it is mentioned in [38] if we consider the space-time as Riemannian instead of Riemann-Cartan, by absorbing the contribution of the torsion of this model into the stress-energy tensor on the rhs of the Einstein equation, then this contribution will act as a source of the Riemannian metric, effectively like an exotic fluid with negative pressure which drives the universe into accelerating expansion. But in the present paper, dark energy is attributed to a scalar field (tachyon) in the framework of teleparallel gravity in which one replaces the Riemannian curvature scalar $R$ by the scalar torsion of teleparallel gravity allowing also a non-minimal coupling between the four-derivative of the scalar field with vector part of torsion tensor.

To study tachyon dynamics more simply let us apply the following transformation (field redefinition) in action (4) [34]

$$
\varphi \rightarrow \phi=\int d \varphi \sqrt{V(\varphi)} \Longleftrightarrow \partial \varphi=\frac{\partial \phi}{\sqrt{V(\phi)}}
$$


It leads to our starting action as follows

$$
S=\int d^{4} x h\left[\frac{T}{2 \kappa^{2}}-V(\phi) \sqrt{1-\frac{\partial_{\mu} \phi \partial^{\mu} \phi}{V(\phi)}}+\frac{\eta f(\phi) \partial_{\mu} \phi \mathcal{V}^{\mu}}{\sqrt{V(\phi)}}\right]+S_{m} .
$$

Considering a spatially-flat FRW metric,

$$
d s^{2}=d t^{2}-a^{2}(t)\left(d r^{2}+r^{2} d \Omega^{2}\right)
$$

and a tetrad choice of the form $h_{\mu}^{a}=\operatorname{diag}(1, a, a, a)$ (which is a solution of gravitational field equation [17]), and a homogeneous scalar field $\phi$ yield to the Friedmann and Raychaudhuri equations as follows,

$$
\begin{gathered}
H^{2}=\frac{\kappa^{2}}{3}\left(\rho_{\phi}+\rho_{m}\right), \\
\dot{H}=-\frac{\kappa^{2}}{2}\left(\rho_{\phi}+P_{\phi}+\rho_{m}+P_{m}\right),
\end{gathered}
$$

where $H=\frac{\dot{a}}{a}$ is the Hubble parameter, $a$ is the scale factor and a dot stands for derivative with respect to the cosmic time $t . \rho_{m}$ and $P_{m}$ are the matter energy density and pressure respectively, satisfying the equation $\dot{\rho}_{m}+3 H\left(1+\omega_{m}\right) \rho_{m}=0$, with $\omega_{m}=\frac{P_{m}}{\rho_{m}}$ the matter equation of state parameter.

The energy momentum tensor associated with the scalar field $\Theta_{a}{ }^{\rho} \equiv-\frac{1}{h} \frac{\delta S_{\phi}}{\delta h^{\alpha}}$ is given by

$$
\begin{gathered}
\Theta_{a}{ }^{\rho}=\eta\left[\frac { f ( \phi ) } { \sqrt { V } } \left(\mathcal{V}^{\rho} \partial_{a} \phi+\nabla_{a} \partial^{\rho} \phi\right.\right. \\
\left.\left.-h_{a}{ }^{\rho} \nabla_{\mu} \partial^{\mu} \phi\right)+\left(\frac{f_{, \phi}}{\sqrt{V}}-\frac{f(\phi) V_{, \phi}}{2 V^{\frac{3}{2}}}\right)\left(\partial_{a} \phi \partial^{\rho} \phi-h_{a}{ }^{\rho} \partial_{\mu} \phi \partial^{\mu} \phi\right)\right] \\
-\mu^{-1} V(\phi) \delta_{a}{ }^{\rho}-\mu \partial_{a} \phi \partial^{\rho} \phi,
\end{gathered}
$$

where $\nabla^{\mu}$ is the covariant derivative in the teleparallel connection $[31,32], f_{, \phi}=\frac{d f(\phi)}{d \phi}, V_{, \phi}=\frac{d V}{d \phi}$ and

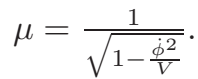

By imposing the FRW metric (7), the energy density and pressure of the scalar field read,

$$
\rho_{\phi}=\mu V(\phi)-3 \eta \frac{f(\phi)}{\sqrt{V}} H \dot{\phi},
$$

and

$$
p_{\phi}=-\mu^{-1} V(\phi)+\eta \dot{\phi}^{2}\left(\frac{f_{, \phi}}{\sqrt{V}}-\frac{f(\phi) V_{, \phi}}{2 V^{\frac{3}{2}}}\right)+\eta \frac{f(\phi)}{\sqrt{V}} \ddot{\phi},
$$

Additionally, variation of the action (6) with respect to the scalar field yields to its evolution equation that in FRW background takes the form

$$
\ddot{\phi}\left[\left(\frac{\partial \mathcal{L}}{\partial X}\right)+(2 X)\left(\frac{\partial^{2} \mathcal{L}}{\partial X^{2}}\right)\right]+\left[3 H\left(\frac{\partial \mathcal{L}}{\partial X}\right)+\dot{\phi}\left(\frac{\partial^{2} \mathcal{L}}{\partial X \partial \phi}\right)\right] \dot{\phi}-\left(\frac{\partial \mathcal{L}}{\partial \phi}\right)=0,
$$

where $X=\frac{1}{2} \dot{\phi}^{2}$.

From equation (13), one can obtain the $\phi$-filed equation of motion more clearly as follows,

$$
\ddot{\phi}+3 \mu^{-2} H \dot{\phi}+\left(1-\frac{3 \dot{\phi}^{2}}{2 V}\right) V_{, \phi}-3 \eta\left(\dot{H}+3 H^{2}\right) \mu^{-3} \frac{f(\phi)}{\sqrt{V}}=0 .
$$

In fact the above equation expresses the energy conservation relation $\dot{\rho}_{\phi}+3 H\left(1+\omega_{\phi}\right) \rho_{\phi}=0$ with $\omega_{\phi}=$ $p_{\phi} / \rho_{\phi}$ the equation of state of the scalar field. 


\section{Dynamical Analysis}

Now we are going to rewrite the evolution equations as a system of autonomous differential equations and study the cosmological properties of the critical points for the system. An autonomous system in general will be of the form $\frac{d \mathbf{Y}}{d \ln a}=f(\mathbf{Y})$, where the column vector $\mathbf{Y}$ is constituted by suitable auxiliary variables and $f(\mathbf{Y})$ is the corresponding column vector of the autonomous equations [35-37].

The critical points $\mathbf{Y}_{c}$ are obtained from $\frac{d \mathbf{Y}}{d \ln a}=0$. In order to study the stability of the equilibrium or critical points we should first expand the system around $\mathbf{Y}_{c}$ as $\mathbf{Y}=\mathbf{Y}_{c}+\mathbf{U}$ where the column vector $\mathbf{U}$ denotes the perturbation of the variables. For each critical point the 1st order perturbation technique lends to the matrix equation $\mathbf{U}^{\prime}=\Sigma$. $\mathbf{U}$ where the matrix $\Sigma$ contains all the coefficients of the perturbation equations. Hence, the stability properties and the type of a specific critical point are determined by the eigenvalues of the matrix $\Sigma$.

(i) If the real part of eigenvalues have opposite signs then the fixed point is a saddle point.

(ii) If three eigenvalues are negative, then the fixed point is stable.

(iii) If the eigenvalues are positive, the fixed point in unstable.

A detailed analysis of the stability criteria is given in Refs [35-37]. A critical point is an attractor in the case (ii) and the universe evolves to the attractor solutions regardless of the initial conditions.

Let us apply the above method to the model (6) by introducing the following new variables:

$$
x \equiv \frac{\dot{\phi}}{\sqrt{V}}, \quad y \equiv \frac{\kappa \sqrt{V}}{\sqrt{3} H}, \quad u \equiv \frac{\kappa \sqrt{f}}{\sqrt{H}}, \quad \alpha \equiv \frac{f_{, \phi}}{\kappa f}, \quad \lambda \equiv-\frac{V_{, \phi}}{\kappa V} .
$$

In terms of these new variables, the field equations can be written as follows,

$$
\begin{aligned}
\frac{d x}{d N}=\left(1-x^{2}\right)\left[(3-s) \eta \mu^{-1} u^{2} y^{-2}+\sqrt{3}(\lambda y-\sqrt{3} x)\right], \\
\frac{d y}{d N}=\left(-\frac{\sqrt{3} \lambda}{2} x y+s\right) y, \\
\frac{d u}{d N}=\frac{1}{2}(\sqrt{3} \alpha x y+s) u, \\
\frac{d \lambda}{d N}=-\sqrt{3} \lambda^{2} x y(\Gamma-1), \\
\frac{d \alpha}{d N}=\sqrt{3} \alpha^{2} x y(\Pi-1),
\end{aligned}
$$

where $N=\ln a$ and the following parameters are defined

$$
\Pi=\frac{f f_{, \phi \phi}}{f_{, \phi}^{2}}, \quad \Gamma=\frac{V V_{, \phi \phi}}{V_{, \phi}^{2}} .
$$

In equation (16)-(18), $s$ is given by

$$
\begin{aligned}
s= & -\frac{\dot{H}}{H^{2}}=3\left(2+\eta^{2} \mu^{-3} u^{4} y^{-2}\right)^{-1} \\
& {\left[\gamma-(\gamma-1)\left(\mu y^{2}-\eta u^{2} x\right)-\mu^{-1} y^{2}+\eta u^{2}\left(-\mu^{-2} x+\frac{\sqrt{3}}{3} y\left(x^{2}(\alpha-\lambda)+\lambda\right)+\eta \mu^{-3} u^{2} y^{-2}\right)\right], }
\end{aligned}
$$

and we mention that $\gamma$ is the barotropic index defined by $\gamma=1+\omega_{m}$ such that $0<\gamma<2$ and also $\mu=\frac{1}{\sqrt{1-x^{2}}}$. The density parameters $\Omega_{i} \equiv\left(\kappa^{2} \rho_{i}\right) /\left(3 H^{2}\right)$ for the scalar field and background matter are given by

$$
\Omega_{\phi}=\mu y^{2}-\eta x u^{2}, \quad \Omega_{m}=1-\Omega_{\phi},
$$


while the equation of state of the field $\omega_{\phi}$ and the effective equation of state can be written as

$$
\omega_{\phi}=\frac{p_{\phi}}{\rho_{\phi}}=\frac{-\mu^{-1} y^{2}+\eta u^{2}\left(-\mu^{-2} x+\frac{\sqrt{3}}{3} y\left(x^{2}(\alpha-\lambda)+\lambda\right)+\frac{1}{3} \eta \mu^{-3}(3-s) u^{2} y^{-2}\right)}{\mu y^{2}-\eta x u^{2}},
$$

and

$$
\begin{aligned}
& \omega_{e f f}=\left(p_{\phi}+p_{m}\right) /\left(\rho_{\phi}+\rho_{m}\right) \\
= & \left(x^{2}-\gamma\right) \mu y^{2}+\eta u^{2}\left(-\mu^{-2} x+\frac{\sqrt{3}}{3} y\left(x^{2}(\alpha-\lambda)+\lambda\right)+\frac{1}{3} \eta \mu^{-3}(3-s) u^{2} y^{-2}\right)+(\gamma-1)\left(1+\eta x u^{2}\right) .
\end{aligned}
$$

Note that the condition for acceleration is $\omega_{e f f}<-1 / 3$.

Once the parameters $\Gamma$ and $\Pi$ are known, equations (16)-(20) become a system of autonomous differential equations and one can study dynamics of the model in a usual way. Considering an exponential potential of the form $V=V_{0} e^{-\lambda \kappa \phi}$ with constant $\lambda$ leads to $\Gamma=1$ and equation (19) can be eliminated from our system of differential equations. In the other hand if we consider a constant $\alpha$ for simplicity then the number of differential equations will be reduced once again. Therefore, we concentrate on a non-minimal coupling function of the form $f(\phi) \propto e^{\beta \phi}$ with a constant $\beta$ which leads to $\Pi=1$ and $\alpha$ becomes a constant. The exponential form of the scalar field potential has been found in higher-order [43] or higher-dimensional gravity theories [44]. In string or Kaluza-Klein type models the moduli fields associated with the geometry of the extra dimensions may have effective exponential potentials due to curvature of the internal spaces, or the interaction of moduli with form fields on the internal spaces. Exponential potentials can also arise due to nonperturbative effects such as gaugino condensation [45]. Significant role of an exponential potential can be seen in various cosmological models (for example see [35]). Moreover, if we consider other forms of $V$ and $f$ the number of autonomous equations will be increased. Extracting the fixed points of a 4 or 5-dimensional autonomous system is pretty complicated. So, by choosing the potential $V=V_{0} e^{-\lambda \kappa \phi}$ and non-minimal coupling function $f(\phi) \propto e^{\beta \phi}$, we have a three dimensional autonomous system (16)-(18).

The critical points of the autonomous system (16)-(18) with constant $\alpha$ and $\lambda$ are listed in Table 1. In the same table we have provided the corresponding values of $\Omega_{\phi}, \omega_{\phi}$ and $\omega_{\text {eff }}$ at each critical point. The conditions needed for existence and acceleration $\left(\omega_{\text {eff }}<-1 / 3\right)$ along with stability properties of the fixed points presented in Table 2. The elements of $3 \times 3$ matrix $\Sigma$ and its three eigenvalues at each critical point are shown in Appendix. Now let us discuss the cosmological behavior and stability properties of the critical points individually.

\section{Critical Point $P_{1}$ :}

This point exists for positive values of $\lambda$ and accelerated expansion of our universe occurs for $\gamma<\frac{2}{3}$. Point $P_{1}$ is stable for $\alpha<-\frac{\lambda}{2}$ and $\lambda>\sqrt{\frac{3 \gamma}{\sqrt{1-\gamma}}}$ and thus attracts the universe at the late-times. Since the dark energy and matter density parameters are at the same order, this point corresponds to a dark energy-dark matter scaling solution, alleviating the cosmological coincidence problem which asks: why are we living in an epoch in which $\Omega_{D E}$ and $\Omega_{D M}$ are comparable? In order to show the above mentioned behavior more transparently, we evolve the autonomous system (16)-(18) numerically for the parameter choices $\lambda=2$ and $\alpha=-1.5$. The phase-space trajectories are depicted in a 3 -dimensional plot in Figure 1 (left panel). It is clear from the figure that in this case the universe is attracted by the stable solution $P_{1}$. An important point about $P_{1}$ is that, it is not a realistic solution in dark energy paradigm due to the presence of the condition $\gamma<\frac{2}{3}$ needed for acceleration. One can solve such a problem by considering a possible coupling between dark energy and dark matter. We will consider this possibility in future works.

\section{Critical Point $P_{2}$ :}

This point is a stable point for $\alpha>-\frac{\lambda}{2}$ and $\lambda<-\sqrt{\frac{3 \gamma}{\sqrt{1-\gamma}}}$ and therefore it can be the late-times state of the universe. Similar to point $P_{1}, P_{2}$ can be accelerated for $\gamma<\frac{2}{3}$ and since $\Omega_{\phi}$ and $\Omega_{m}$ are of the same order 
it is a scaling attractor (it can help to alleviate the cosmological coincidence problem).

Depending on the background matter (i.e. the values of $\gamma$ ) the dark energy equation of state lies in the quintessence regime $\left(\omega_{\phi}>-1\right)$ or is equal to cosmological constant value $\left(\omega_{\phi}=-1\right)$. In Figure 1 (right panel) the phase-space trajectories of the system (16)-(18) for the model parameter values $\lambda=-2, \alpha=1.5$ and $\eta=\frac{1}{2}$ are presented. In this case the attractor point of the system is $P_{2}$ as it is clear from the figure. Once again note that $P_{2}$ is also a non-realistic solution because of the condition $\gamma<\frac{2}{3}$ which is needed for acceleration.

\section{Critical Point $P_{3}$ :}

The fixed point $P_{3}$ corresponds to a dark energy dominated de Sitter solution with $\Omega_{\phi}=1$ and $\omega_{e f f}=-1$. This point exists when $\frac{\lambda}{\eta}<0$ and can be accelerated for all values. It is an attractor solution of the model for $\lambda>0, \alpha>-\frac{\lambda}{2}$ or $\lambda<0, \alpha<-\frac{\lambda}{2}$. Such a behavior of the system (16)-(18) are depicted in Figure 2 for the choices $\lambda=2, \alpha=1$ and $\eta=-\frac{1}{2}$ (left panel) and $\lambda=-2, \alpha=0.5$ and $\eta=\frac{1}{2}$ (right panel) respectively.

\section{Critical Point $P_{4}$ :}

At this point $\Omega_{\phi}$ and $\Omega_{m}$ are of the same order and thus it can help to alleviate the cosmological coincidence problem. $P_{4}$ exists for positive values of $\lambda$ and universe at this point can be accelerated for the conditions presented in Table 2. However, since the eigenvalues of the $3 \times 3$ matrix $\Sigma$ of the corresponding linearized perturbation equations at this point are very complicated, we can not conclude about its stability analytically. However we evolved the system numerically and find that as long as $\alpha<-\frac{\lambda}{2}$ this point can be a stable point as it is shown in Figure 3. In this figure the parameters of the model have the values $\lambda=3$, $\alpha=-2$ and $\eta=\frac{1}{2}$.

\section{Critical Point $P_{5}$ :}

This critical point has the same acceleration and eigenvalues properties as point $P_{4}$ but it exists for negative values of $\lambda$. Our numerical computations reveal that $P_{5}$ could not be a stable solution of the model. The cosmological evolutions of the density fraction parameters $\Omega_{\phi}$ and $\Omega_{m}$ are also shown in Figure 4 . Before closing this section, let us make comments on the results of the past models with a non-minimally coupled scalar field in the framework of teleparallel gravity. Dynamics of teleparallel dark energy in which a canonical scalar field (quintessence) non-minimally coupled to torsion scalar was first studied by Wei in [27]. The Author has considered the interaction between dark energy and dark matter and for two kinds of potentials has obtained some attractor points. But no scaling attractor was found. Xu et al. [26] have investigated phase space analysis of the model using a new set of dimensionless variables. Although they have found a dark energy dominated solution with $\omega=-1$, unfortunately at this point $\Omega_{\phi}=1$ and so it is not a scaling attractor of the model.

Finally a scaling attractor of teleparallel dark energy model has been found in Ref. [28]. The coupling function between scalar field and torsion scalar was generalized to a arbitrary function $f(\phi)$ and interaction between dark energy and dark matter was also considered.

Furthermore, dynamics of tachyonic teleparallel dark energy has been studied in [30] and although scaling solutions in this model have been found, they were not stable solutions. In the other hand stable solutions in tachyonic teleparallel dark energy are not scaling attractors of the model. Phase-space analysis of a new teleparallel dark energy model has been done in [33]. In such a model instead of tachyon, quintessence plays the role of dark energy in action (4). A de Sitter attractor solution was found in the case that the non-minimal coupling function is of the form $f(\phi) \propto \phi$ and a attractor solution was found for general form of $f(\phi)$. But no scaling attractor was presented in the model. In the present paper we have utilized a non-canonical scalar field (tachyon) in action (4) and extracted the fixed points of the model. In addition to dark energy dominated solution $\left(P_{3}\right)$ that accelerates the universe, we have obtained a scaling attractor solution $\left(P_{4}\right)$ that fulfills conditions required for current state of our universe.

\section{Conclusion}

According to observational data we are living in an epoch in which the densities of dark energy and dark matter are comparable [4], although they scale differently during the expansion history of our universe. This 
Table 1: The critical points of the autonomous system (16)-(18) for constant $\alpha$ and the corresponding values of the dark energy density parameter $\Omega_{\phi}$, the dark energy equation of state parameter $\omega_{\phi}$ and the effective equation of state parameter $\omega_{\text {eff }}$. We use the notation $A=-\lambda^{2}+\sqrt{\lambda^{4}+36}$.

\begin{tabular}{|c|c|c|c|c|c|c|}
\hline Name & $x_{c}$ & $y_{c}$ & $u_{c}$ & $\Omega_{\phi}$ & $\omega_{\phi}$ & $\omega_{\text {eff }}$ \\
\hline$P_{1}$ & $\sqrt{\gamma}$ & $\frac{\sqrt{3 \gamma}}{\lambda}$ & 0 & $\frac{3 \gamma}{\lambda^{2} \sqrt{1-\gamma}}$ & $\gamma-1$ & $\gamma-1$ \\
\hline$P_{2}$ & $-\sqrt{\gamma}$ & $-\frac{\sqrt{3 \gamma}}{\lambda}$ & 0 & $\frac{3 \gamma}{\lambda^{2} \sqrt{1-\gamma}}$ & $\gamma-1$ & $\gamma-1$ \\
\hline$P_{3}$ & 0 & 1 & $\sqrt{\frac{-\sqrt{3} \lambda}{3 \eta}}$ & 1 & -1 & -1 \\
\hline$P_{4}$ & $\frac{\sqrt{2}}{6} \lambda \sqrt{A}$ & $\frac{\sqrt{6}}{6} \sqrt{A}$ & 0 & $\frac{1}{6} \frac{A}{\sqrt{1-\frac{\lambda^{2}}{18}}}$ & $\frac{\lambda^{2}}{18} A-1$ & $\frac{1}{6} \frac{A\left(\frac{\lambda^{2}}{18} A-\gamma\right)}{\sqrt{1-\frac{\lambda^{2}}{18} A}}+\gamma-1$ \\
\hline$P_{5}$ & $-\frac{\sqrt{2}}{6} \lambda \sqrt{A}$ & $\frac{\sqrt{6}}{6} \sqrt{A}$ & 0 & $\frac{1}{6} \frac{A}{\sqrt{1-\frac{\lambda^{2}}{18} A}}$ & $\frac{\lambda^{2}}{18} A-1$ & $\frac{1}{6} \frac{A\left(\frac{\lambda^{2}}{18} A-\gamma\right)}{\sqrt{1-\frac{\lambda^{2}}{18} A}}+\gamma-1$ \\
\hline
\end{tabular}

cosmological coincidence problem can be alleviated in most dark energy models via the method of scaling attractors that correspond to accelerating universe and ratio $\frac{\Omega_{\phi}}{\Omega_{m}}$ of order 1 . If these conditions are fulfilled, then the universe will result to that solution at late-times, independently of the initial conditions and the basic observational requirement will be satisfied [42].

In the present paper, motivated by the recent work of Otalora [33], we proposed a new model of dark energy in which the four-derivative of tachyon field is non-minimally coupled to the vector part of torsion tensor. As it is mentioned in [33], such a non-minimal coupling has no analogue in general relativity, because in general relativity decomposition of gravitational field in a form analogous to the decomposition of torsion in teleparallel gravity, is not possible.

We studied the dynamical behavior of our model in details. In order to reduce the number of autonomous equations, we have chosen an exponential tachyonic potential $V=V_{0} e^{-\lambda \kappa \phi}$ and a non-minimal coupling function of the form $f(\phi) \propto e^{\beta \phi}$. These choices leave a three dimensional autonomous equations out of the field equations (8), (9) and (14). We found five critical points presented in Table 1. Points $P_{1}$ and $P_{2}$ are scaling attractors of the model for suitable choices of the model parameters as shown in Table 2 and depicted in Figure 1. However these points have a disadvantage that acceleration occurs for $\gamma<\frac{2}{3}$ which makes them non-realistic points in applying to dark energy.

Point $P_{3}$ corresponds to a completely dark energy dominated solution that can be accelerated but it is not a scaling attractor of the model because at this point $\Omega_{\phi}=1$. The equation of state at this point behaves like a cosmological constant regardless of the values of the model parameters. The most interesting critical point is the point $P_{4}$ which is a scaling attractor that can be accelerated for the conditions presented in Table 2. Thus point $P_{4}$ can help to alleviate the cosmological coincidence problem and fulfills requirement according to cosmological observations. Here we mention that in Ref [33] where quintessence scalar field plays the role of dark energy, there is no scaling attractor that can be accelerated. In comparison to tachyonic teleparallel dark energy [46, 47], once again we emphasize that in our previous works tachyon field non-minimally coupled to the torsion scalar but here the field non-minimally coupled to the vector torsion. The significant advantage of the present model is that while in the previous models [46, 47] there was no scaling attractor here we obtain a scaling attractor without considering an extra term such as interaction between dark energy and dark matter as usually done in the literature to obtain scaling attractors. Therefore, Point $P_{4}$ makes an important difference between our model and the models proposed in $[33,46,47]$. And finally point $P_{5}$ is not a stable solution of the system (16)-(18). Before closing this section we mention that besides the model (6), generalized tachyon field in the context of teleparallel gravity also represents scaling attractors [48]. Thus, in order to obtain a scaling attractor through tachyonic teleparallel dark energy model we should either generalized tachyon action [48] or consider non-minimal coupling of tachyon field with vector torsion. Further study of our model can be done for other scalar field potentials $V(\phi)$ and coupling function $f(\phi)$. 
Table 2: Existence, acceleration and stability conditions of the fixed points in Table 1.

\begin{tabular}{|c|c|c|c|}
\hline label & existence & acceleration & stability \\
\hline \multirow{2}{*}{$P_{1}$} & \multirow{2}{*}{$\lambda>0$} & \multirow{2}{*}{$\gamma<\frac{2}{3}$} & $\begin{array}{r}\text { stable point if } \\
\alpha<-\frac{\lambda}{2} \text { and } \lambda>\sqrt{\frac{3 \gamma}{\sqrt{1-\gamma}}}\end{array}$ \\
\hline & & & $\begin{array}{c}\text { saddle point if } \\
\alpha>-\frac{\lambda}{2} \text { or } \lambda<\sqrt{\frac{3 \gamma}{\sqrt{1-\gamma}}}\end{array}$ \\
\hline \multirow{2}{*}{$P_{2}$} & \multirow{2}{*}{$\lambda<0$} & \multirow{2}{*}{$\gamma<\frac{2}{3}$} & $\begin{array}{c}\text { stable point if } \\
\alpha>-\frac{\lambda}{2} \text { and } \lambda<-\sqrt{\frac{3 \gamma}{\sqrt{1-\gamma}}}\end{array}$ \\
\hline & & & $\begin{array}{c}\text { saddle point if } \\
\alpha<-\frac{\lambda}{2} \text { or } \lambda>-\sqrt{\frac{3 \gamma}{\sqrt{1-\gamma}}}\end{array}$ \\
\hline \multirow{2}{*}{$P_{3}$} & \multirow{2}{*}{$\frac{\lambda}{\eta}<0$} & \multirow{2}{*}{ all values } & $\begin{array}{c}\text { stable point if } \\
\lambda>0 \text { and } \alpha>-\frac{\lambda}{2} \\
\text { or } \\
\lambda<0 \text { and } \alpha<-\frac{\lambda}{2} \\
\end{array}$ \\
\hline & & & $\begin{array}{c}\text { saddle point if } \\
\lambda<0 \text { and } \alpha>-\frac{\lambda}{2} \\
\text { or } \\
\lambda>0 \text { and } \alpha<-\frac{\lambda}{2}\end{array}$ \\
\hline \multirow{2}{*}{$P_{4}$} & \multirow{2}{*}{$\lambda>0$} & $\gamma<\frac{2\left(2 \sqrt{1-\frac{\lambda^{2}}{18} A}-\frac{\lambda^{2} A^{2}}{36}\right)}{2}$ & \multirow{2}{*}{ see explanations about this point in the text } \\
\hline & & $6 \sqrt{1-\frac{\lambda^{2}}{18} A}-A$ & \\
\hline \multirow{2}{*}{$P_{5}$} & \multirow{2}{*}{$\lambda<0$} & $\gamma<\frac{2\left(2 \sqrt{1-\frac{\lambda^{2}}{18} A}-\frac{\lambda^{2} A^{2}}{36}\right)}{3}$ & \multirow{2}{*}{ see explanations about this point in the text } \\
\hline & & $\gamma<\longdiv { 6 \sqrt { 1 - \frac { \lambda ^ { 2 } } { 1 8 } A } - A }$ & \\
\hline
\end{tabular}



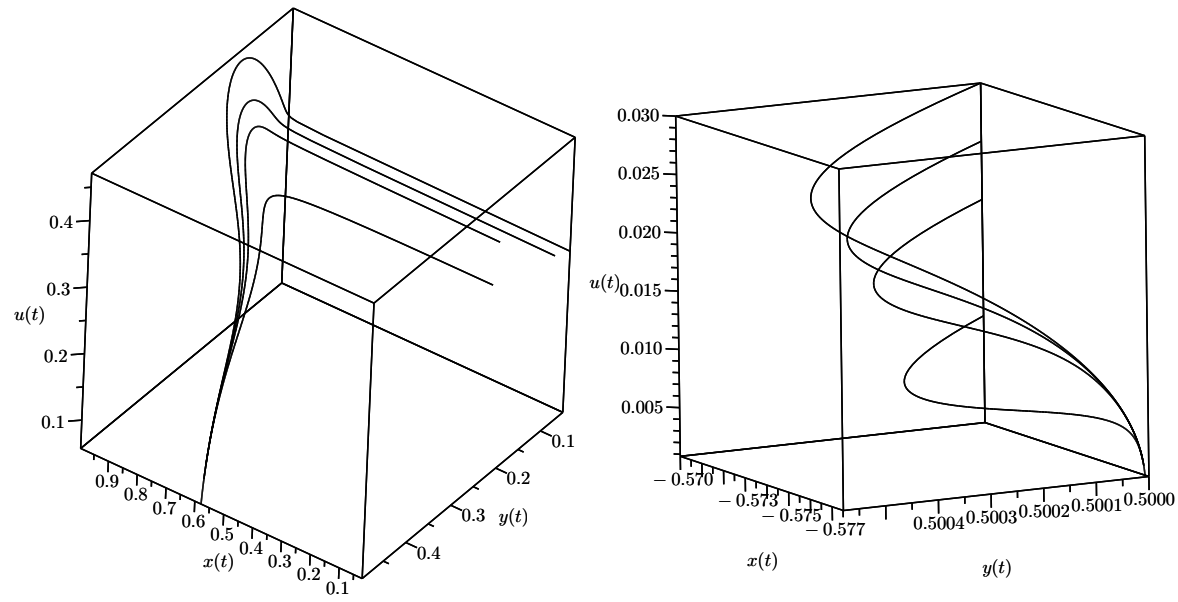

Figure 1: 3-dimensional phase-space trajectories of the cosmological scenario (16)-(18) with stable attractor $P_{1}$ (left) for the parameter choices $\lambda=2, \alpha=-1.5$ and $\eta=\frac{1}{2}$ and stable attractor $P_{2}$ (right) for $\lambda=-2, \alpha=1.5$ and $\eta=\frac{1}{2}$.
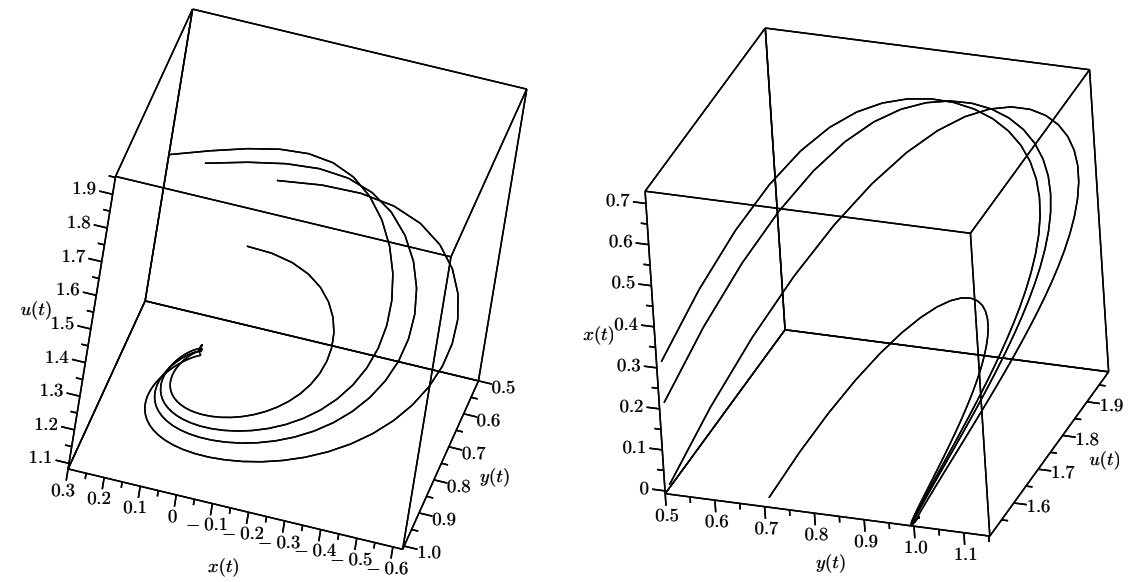

Figure 2: 3-dimensional phase-space trajectories of the cosmological scenario (16)-(18) with stable attractor $P_{3}$ for $\lambda=2, \alpha=1$ and $\eta=-\frac{1}{2}$ (left) and $\lambda=-2, \alpha=0.5$ and $\eta=\frac{1}{2}$ (right). 


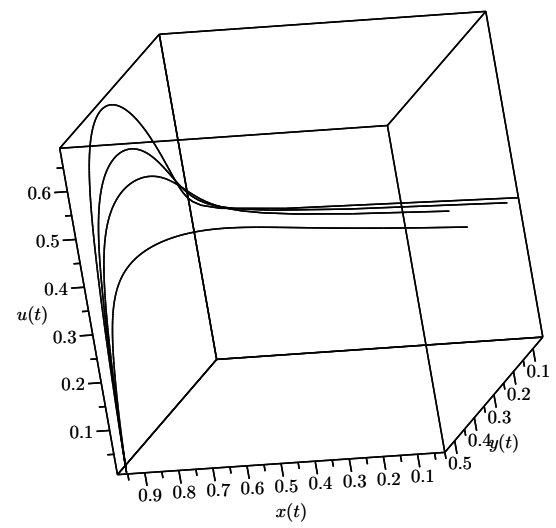

Figure 3: 3-dimensional phase-space trajectories of the cosmological scenario (16)-(18) with stable attractor $P_{4}$ for $\lambda=3, \alpha=-2$ and $\eta=\frac{1}{2}$.

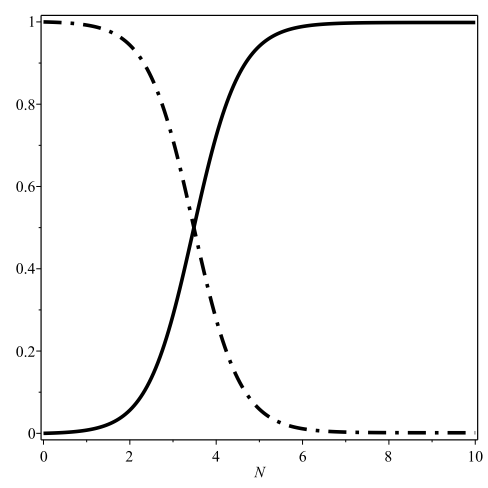

Figure 4: Evolution of $\Omega_{\phi}$ (solid) and $\Omega_{m}$ (dotdashed) with $\lambda=0.3, \alpha=-1.5$ and $\eta=\frac{1}{2}$. The initial conditions are $x_{i}=10^{-8}, y_{i}=3.7 \times 10^{-2}$ and $u_{i}=10^{-7}$. The corresponding values of $\Omega_{\phi}$ and $\Omega_{m}$ at the present epoch $(N \simeq 4)$ are $\Omega_{\phi} \approx 0.72$ and $\Omega_{m} \approx 0.28$. 


\section{Appendix: Elements of perturbation matrix $\Sigma$ and its eigenval- ues at critical points}

The components of matrix $\Sigma$ which represents the coefficients of the linearized perturbation equations are given by

$$
\begin{aligned}
& \Sigma_{11}=-2 x_{c}\left[\left(3-s_{c}\right) \eta \mu_{c}^{-1} u_{c}^{2} y_{c}^{-2}+\sqrt{3}\left(\lambda y_{c}-\sqrt{3} x_{c}\right)\right]-3 \mu_{c}^{-3} \eta u_{c}^{2} y_{c}^{-2}\left(2+\eta^{2} \mu_{c}^{-3} u_{c}^{4} y_{c}^{-2}\right)^{-1} \\
& {\left[s_{c} \mu_{c}^{-1} \eta^{2} x_{c} u_{c}^{4} y_{c}^{-2}-(\gamma-1)\left(\mu_{c}^{3} x_{c} y_{c}^{2}-\eta u_{c}^{2}\right)+\mu_{c} x_{c} y_{c}^{2}+\eta u_{c}^{2}\left(3 x_{c}^{2}-1+\frac{2 \sqrt{3}}{3} x_{c} y_{c}(\alpha-\lambda)-3 \mu_{c}^{-1} \eta x_{c} u_{c}^{2} y_{c}^{-2}\right)\right]} \\
& -\mu_{c}^{-1} \eta x_{c} u_{c}^{2} y_{c}^{-2}\left(3-s_{c}\right)-3 \mu_{c}^{-2} \\
& \Sigma_{12}=\sqrt{3} \lambda\left(1-x_{c}^{2}\right)-2 \eta \mu_{c}^{-3} u_{c}^{2} y_{c}^{-3}-\eta \mu_{c}^{-3} u_{c}^{2} y_{c}^{-2} \\
& \left(2+\eta^{2} \mu_{c}^{-3} u_{c}^{4} y_{c}^{-2}\right)^{-1}\left[2 \eta^{2} \mu_{c}^{-3} s_{c} u_{c}^{4} y_{c}^{-3}+6 \mu_{c} y_{c}\left(x_{c}^{2}-\gamma\right)+\eta u_{c}^{2}\left(\sqrt{3}\left(x_{c}^{2}(\alpha-\lambda)+\lambda\right)+6 \eta \mu_{c}^{-3} u_{c}^{2} y_{c}^{-3}\right)\right] \\
& \Sigma_{13}=2\left(3-s_{c}\right) \eta \mu_{c}^{-3} u_{c} y_{c}^{-2}-2 \eta \mu_{c}^{-3} u_{c}^{2} y_{c}^{-2}\left(2+\eta^{2} \mu_{c}^{-3} u_{c}^{4} y_{c}^{-2}\right)^{-1} \\
& {\left[3 \eta u_{c}\left((\gamma-1) x_{c}-\mu_{c}^{-2} x_{c}+\frac{\sqrt{3}}{3} y_{c}\left(x_{c}^{2}(\alpha-\lambda)+\lambda\right)+2 \eta \mu_{c}^{-3} u_{c}^{2} y_{c}^{-2}\right)-2 \eta^{2} \mu_{c}^{-3} s_{c} u_{c}^{3} y_{c}^{-2}\right],} \\
& \Sigma_{21}=-\frac{\sqrt{3}}{2} \lambda y_{c}^{2}-3 y_{c}\left(2+\eta^{2} \mu_{c}^{-3} u_{c}^{4} y_{c}^{-2}\right)^{-1} \\
& {\left[s_{c} \mu_{c}^{-1} \eta^{2} x_{c} u_{c}^{4} y_{c}^{-2}-(\gamma-1)\left(\mu_{c}^{3} x_{c} y_{c}^{2}-\eta u_{c}^{2}\right)+\mu_{c} x_{c} y_{c}^{2}+\eta u_{c}^{2}\left(3 x_{c}^{2}-1+\frac{2 \sqrt{3}}{3} x_{c} y_{c}(\alpha-\lambda)-3 \mu_{c}^{-1} \eta x_{c} u_{c}^{2} y_{c}^{-2}\right)\right]}
\end{aligned}
$$

$$
\begin{aligned}
& \Sigma_{22}=s_{c}-\sqrt{3} \lambda x_{c} y_{c}+y_{c}\left(2+\eta^{2} \mu_{c}^{-3} u_{c}^{4} y_{c}^{-2}\right)^{-1} \\
& {\left[2 \eta^{2} \mu_{c}^{-3} s_{c} u_{c}^{4} y_{c}^{-3}+6 \mu_{c} y_{c}\left(x_{c}^{2}-\gamma\right)+\eta u_{c}^{2}\left(\sqrt{3}\left(x_{c}^{2}(\alpha-\lambda)+\lambda\right)+6 \eta \mu_{c}^{-3} u_{c}^{2} y_{c}^{-3}\right)\right], \quad(30)} \\
& \Sigma_{23}=2 y_{c}\left(2+\eta^{2} \mu_{c}^{-3} u_{c}^{4} y_{c}^{-2}\right)^{-1} \\
& \left.\left[3 \eta u_{c}\left((\gamma-1) x_{c}-\mu_{c}^{-2} x_{c}+\frac{\sqrt{3}}{3} y_{c}\left(x_{c}^{2}(\alpha-\lambda)+\lambda\right)+2 \eta \mu_{c}^{-3} u_{c}^{2} y_{c}^{-2}\right)-2 \eta^{2} \mu_{c}^{-3} s_{c} u_{c}^{3} y_{c}^{-2}\right], \quad(31)\right] \\
& \Sigma_{31}=\frac{\sqrt{3}}{2} \alpha y_{c} u_{c}-\frac{3}{2} u_{c}\left(2+\eta^{2} \mu_{c}^{-3} u_{c}^{4} y_{c}^{-2}\right)^{-1} \\
& {\left[s_{c} \mu_{c}^{-1} \eta^{2} x_{c} u_{c}^{4} y_{c}^{-2}-(\gamma-1)\left(\mu_{c}^{3} x_{c} y_{c}^{2}-\eta u_{c}^{2}\right)+\mu_{c} x_{c} y_{c}^{2}+\eta u_{c}^{2}\left(3 x_{c}^{2}-1+\frac{2 \sqrt{3}}{3} x_{c} y_{c}(\alpha-\lambda)-3 \mu_{c}^{-1} \eta x_{c} u_{c}^{2} y_{c}^{-2}\right)\right],}
\end{aligned}
$$




$$
\begin{aligned}
\Sigma_{32}=\frac{\sqrt{3}}{2} \alpha x_{c} u_{c}+\frac{1}{2} u_{c}\left(2+\eta^{2} \mu_{c}^{-3} u_{c}^{4} y_{c}^{-2}\right)^{-1} \\
{\left[2 \eta^{2} \mu_{c}^{-3} s_{c} u_{c}^{4} y_{c}^{-3}+6 \mu_{c} y_{c}\left(x_{c}^{2}-\gamma\right)+\eta u_{c}^{2}\left(\sqrt{3}\left(x_{c}^{2}(\alpha-\lambda)+\lambda\right)+6 \eta \mu_{c}^{-3} u_{c}^{2} y_{c}^{-3}\right)\right], } \\
\Sigma_{33}=\frac{1}{2}\left(\sqrt{3} \alpha x_{c} y_{c}+s_{c}\right)+\frac{1}{2} u_{c}\left(2+\eta^{2} \mu_{c}^{-3} u_{c}^{4} y_{c}^{-2}\right)^{-1} \\
\quad\left[3 \eta u_{c}\left((\gamma-1) x_{c}-\mu_{c}^{-2} x_{c}+\frac{\sqrt{3}}{3} y_{c}\left(x_{c}^{2}(\alpha-\lambda)+\lambda\right)+2 \eta \mu_{c}^{-3} u_{c}^{2} y_{c}^{-2}\right)-2 \eta^{2} \mu_{c}^{-3} s_{c} u_{c}^{3} y_{c}^{-2}\right] .
\end{aligned}
$$

In the above expressions by $s_{c}$ and $\mu_{c}$ we mean evaluation of them at critical points i.e

$$
\mu_{c}=1 / \sqrt{1-x_{c}^{2}}
$$

and

$$
\begin{aligned}
& s_{c}=3\left(2+\eta^{2} \mu_{c}^{-3} u_{c}^{4} y_{c}^{-2}\right)^{-1} \\
& {\left[\gamma-(\gamma-1)\left(\mu_{c} y_{c}^{2}-\eta u_{c}^{2} x_{c}\right)-\mu_{c}^{-1} y_{c}^{2}+\eta u_{c}^{2}\left(-\mu_{c}^{-2} x_{c}+\frac{\sqrt{3}}{3} y_{c}\left(x_{c}^{2}(\alpha-\lambda)+\lambda\right)+\eta \mu_{c}^{-3} u_{c}^{2} y_{c}^{-2}\right)\right] .}
\end{aligned}
$$

Although elements of $\Sigma$ seem complicated, inserting the explicit critical points shown in Table 1, the matrix $\Sigma$ acquires a simple form that allows for calculation of its eigenvalues. The eigenvalues of the matrix $\Sigma$ (namely $\nu_{1}, \nu_{2}, \nu_{3}$ ), for each critical point are as follows:

points $P_{1}$ and $P_{2}$ :

$$
\nu_{1}=\frac{3 \gamma(\lambda+2 \alpha)}{4 \lambda}, \quad \nu_{2,3}=\frac{3\left(\gamma \lambda-2 \lambda \pm \sqrt{17 \gamma^{2} \lambda^{2}-20 \gamma \lambda^{2}+4 \lambda^{2}+48 \gamma^{2} \sqrt{1-\gamma}}\right)}{4 \lambda}
$$

Point $P_{3}$ :

$$
\nu_{1,2}=\frac{-3\left(\lambda^{2}+6 \pm \sqrt{-3 \lambda^{4}-12 \lambda^{2}+36-48 \lambda \alpha-8 \alpha \lambda^{3}}\right)}{2\left(6+\lambda^{2}\right)}, \quad \nu_{3}=-3 \gamma
$$

\section{References}

[1] E. J. Copeland, M. Sami and S. Tsujikawa, Int. J. Mod. Phys. D 15, 1753 (2006); V. Sahni and A. Starobinsky, Int. J. Mod. Phys. D 9, 373-444 (2000); V. Sahni and A. Starobinsky, Int. J. Mod. Phys. D 15, 2105-2132 (2006).

[2] Y. -F. Cai, E. N. Saridakis, M. R. Setare and J. -Q. Xia, Phys. Rept. 493, 1 (2010).

[3] Riess et al., Astron. J. 116, 1009 (1998); Perlmutter et al., Astrophys. J., 517, 565 (1999).

[4] J. Frieman, M. Turner and D. Huterer, Ann. Rev. Astron. Astrophys. 46, 385 (2008); M. Li, X.D. Li, S. Wang and Y. Wang, Commun. Theor. Phys. 56, 525 (2011); K. Bamba, S. Capozziello, S. Nojiri and S. D. Odintsov, Astrophys. Space Sci. 342, 155-228 (2012).

[5] V. Sahni, astro-ph/0403324; J. Martin, Mod. Phys. Lett. A 23, 1252 (2008); S. Tsujikawa, Class. Quant. Grav. 30, 21400 (2013).

[6] V. Sahni and S. Habib, Phys. Rev. Lett. 81, 1766 (1998); J. P. Uzan, Phys. Rev. D 59, 123510 (1999); N. Bartolo and M. Pietroni, Phys. Rev. D 61, 023518 (2000). 
[7] E. Elizalde, S. Nojiri and S. D. Odintsov, Phys. Rev. D 70, 043539 (2004).

[8] O. Hrycyna and M. Szydlowski, JCAP 0904, 026 (2009); O. Hrycyna and M. Szydlowski, Phys. Rev. $D$ 76, 123510 (2007).

[9] C. Armendariz-Picon and E. A. Lim, JCAP 0508, 007 (2005); D. Bertacca, N. Bartolo and S. Matarrese, JCAP 0805, 005 (2008).

[10] T. Chiba, T. Okabe, M. Yamaguchi, Phys. Rev. D 62, 023511 (2000); C. Armendariz-Picon, V. Mukhanov and P. J. Steinhardt, Phys. Rev. Lett. 85, 4438 (2000); C. Armendariz-Picon, V. Mukhanov and P. J. Steinhardt, Phys. Rev. D 63, 103510 (2001).

[11] D. A. Steer and F. Vernizzi, Phys. Rev. D 70, 043527 (2004).

[12] S. Campo, R. Herrera and A. Toloza, Phys. Rev. D 79, 083507 (2009).

[13] V. Mukhanov and A. Vikman, JCAP 0602, 004 (2006); G. Panotopoulos, Phys. Rev. D 76, 127302 (2007).

[14] S. Alexander, Phys. Rev. D 65, 023507 (2002); A. Mazumdar, S. Panda and A. Perez-Lorenzana, Nucl. Phys. B 614, 101 (2001); G. Gibbons, Phys. Lett. B 537, 1 (2002).

[15] M.R. Garousi, M. Sami and S. Tsujikawa, Phys. Rev. D 71, 083005 (2005); E.J. Copeland, M.R. Garousi, M. Sami and S. Tsujikawa, Phys. Rev. D 71, 043003 (2005); A. Sen, JHEP 9910, 008 (1999); E. Bergshoeff, M. de Roo, T. de Wit, E. Eyras and S. Panda, JHEP 0005, 009 (2000); J. Kluson, Phys. Rev. D 62, 126003 (2000).

[16] A. Einstein, Sitzungsber. Preuss. Akad. Wiss. Phys. Math. Kl., 217 (1928); 224 (1928); A. Einstein, Math. Ann. 102, 685 (1930); For English translation, see A. Unzicker and T. Case, arXiv:physics/0503046 12 .

[17] K. Hayashi and T. Shirafuji, Phys. Rev. D 19, 3524 (1979); Addendum-ibid. D 24, 3312 (1981).

[18] G. R. Bengochea and R. Ferraro, Phys. Rev. D 79, 124019 (2009).

[19] E. V. Linder, Phys. Rev. D 81, 127301 (2010); Erratum-ibid. D 82, 109902.

[20] R. J. Yang, Eur. Phys. J. 71, 1797 (2011); T. Wang, Phys. Rev. D 84, 024042 (2011).

[21] R. Ferraro and F. Fiorini, Phys. Rev. D 84, 083518 (2011); C. G. Boehmer, A. Mussa and N. Tamanini, Class. Quant. Grav. 28, 245020 (2011).

[22] M. Sharif and S. Rani, Mod. Phys. Lett. A 26, 1657 (2011); R. Myrzakulov, Eur. Phys. J. C 71, 1752 (2011).

[23] B. Li, T. P. Sotiriou and J. D. Barrow, Phys. Rev. D 83, 064035 (2011); T. P. Sotiriou, B. Li and J. D. Barrow, Phys. Rev. D 83, 104030 (2011).

[24] C. -Q. Geng, C. -C. Lee, E. N. Saridakis, Y. -P. Wu, Phys. Lett. B 704, 384-387 (2011).

[25] C. -Q. Geng, C. -C. Lee and E. N. Saridakis, JCAP 1201, 002 (2012).

[26] C. Xu, E. N. Saridakis and G. Leon, arXiv:1202.3781 [gr-qc]].

[27] H. Wei, Phys. Lett. B 712, 430 (2012).

[28] G. Otalora, JCAP 1307, 044 (2013).

[29] A. Banijamali and B. Fazlpour, Astrophys. Space Scie. 342, 229 (2012). 
[30] G. Otalora, Phys. Rev. D 88, 063505 (2013).

[31] R. Aldrovandi and J. G. Pereira, Teleparallel Gravity: An Introduction (Springer, Dordrecht, 2013 ); J. G. Pereira, AIP Conf. Proc. 1483 (2012) 239-259, arXiv:1210.0379; J. G. Pereira, Teleparallelism: a new insight into gravitation, in Springer Handbook of Spacetime, ed. by A. Ashtekar and V. Petkov (Springer, Dordrecht, 2013) arXiv:1302.6983.

[32] H. I. Arcos and J. G. Pereira, Int. J. Mod. Phys. D 13, 2193 (2004).

[33] G. Otalora, arXiv:1402.2256 [gr-qc]].

[34] I. Quiros, T. Gonzalez, D. Gonzalez, Y. Napoles, R. Garcia-Salcedo and C. Moreno, Class. Quant. Grav. 27, 215021 (2010).

[35] E. J. Copeland, A. R. Liddle and D. Wands, Phys. Rev. D 57, 4686-4690 (1998).

[36] D. J. Holden and D. Wands, Phys. Rev. D 61, 043506 (2000).

[37] S. L. Ross, Differential Equations, 3rd ed. (John Wiley and Sons), 2004.

[38] K-F. Shie, J. M. Nester and H-J. Yo, Phys. Rev. D. 78, 023522 (2008).

[39] V. Faraoni, Phys. Rev. D 62, 023504 (2000).

[40] Q. Shafi and C. Wetterich, Phys. Lett. B 152, 51 (1985).

[41] Q. Shafi and C. Wetterich, Nucl. Phys. B 289, 787 (1987).

[42] X. Chen, Y. Gong and E. N. Saridakis, JCAP 0904, 001 (2009).

[43] B. Whitt, Phys. Lett. B 145, 176 (1984).

[44] M. B. Green, J. H. Schwarz and E. Witten, Superstring Theory, Cambridge University Press (1987).

[45] B. de Carlos, J. A. Casas and C. Munoz, Nucl. Phys. B 399, 623 (1993).

[46] B. Fazlpour and A. Banijamali, Adv. High Energy Phys. 2013, 279768 (2013).

[47] A. Banijamali, Adv. High Energy Phys. 2014, 631630 (2014).

[48] B. Fazlpour and A. Banijamali, Adv. High Energy Phys. 2015, 283273 (2015). 